\title{
What is life? The emergence of life in a mineral world
}

\author{
Frances Westall ${ }^{1}$, Thomas Georgelin ${ }^{2}$, Keyron Hickman-Lewis ${ }^{1}$, Avinash Dass ${ }^{1}$, \\ Terrence Kee ${ }^{3}$, André Brack ${ }^{1}$ \\ ${ }^{1}$ Centre de Biophysique Moléculaire, UPR CNRS4301, Orléans, France \\ ${ }^{2}$ Sorbonne Universités, UPMC Paris 06, CNRS UMR 7197, Laboratoire de Réactivité de Surface \\ ${ }^{3}$ School of Chemistry, University of Leeds, UK \\ frances.westall@cnrs.fr
}

\begin{abstract}
The premise of this talk is that, apart from liquid water and carbon molecules, specific environmental components and conditions were essential for the origin of life, i.e. phosphate, reactive rocks and minerals bathed by warm to hot hydrothermal fluids. The rocksand minerals provided reactive substrates producing energy to fuel reactions creating the prebiotic building bricks of life. They also promoted concentration of organic molecules and contributed to their conformation, stabilisation and the complexification of larger, stable molecules. These reactions took place under environmental conditions very different to those of the Earth today: anoxygenic, warm to hot and acidic ocean water, much volcanic and hydrothermal activity, high flux of UV radiation....However, these are conditions probably common on any rocky planet hosting water. Primitive life forms, such as chemotrophs, could be the most common inhabitants of the Universe but are also the most difficult to detect. Robotic exploration may help mankind explore beyond the limits of human existence and search for life in other stellar systems.
\end{abstract}

